\title{
$\mathrm{CD}^{+} 0^{+}$Anaplastic Large-Cell Lymphoma With Aberrant Expression of CD13: Case Report and Review of the Literature

\author{
Cherie H. Dunphy, ${ }^{1 *}$ Laura J. Gardner, ${ }^{1}$ Jose L. Manes, ${ }^{2}$ Christopher S. Bee, ${ }^{1}$ \\ and Kutay Taysi ${ }^{3}$ \\ ${ }^{1}$ Division of Hematopathology, Department of Pathology, St. Louis University Health Science Center, \\ St. Louis, Missouri \\ ${ }^{2}$ Department of Pathology, St. Luke's Medical Center, Chesterfield, Missouri, \\ ${ }^{3}$ Cytogenetics Laboratory, St. John's Medical Center, St. Louis, Missouri
}

\begin{abstract}
CD13 is commonly expressed in hematopoietic malignancies of myelomonocytic origin and has less commonly been described in lymphoid neoplasms, including acute lymphoblastic leukemia, B-cell lymphoproliferative disorders, and plasma cell malignancies. Aberrant CD13 expression has rarely been described in KP-1 (CD68)-positive large-cell lymphomas. However, CD13 positivity has not previously been described in a case of $\mathrm{CD}_{30} 0^{+}\left(\mathrm{ALK}-1^{+}\right)$anaplastic largecell lymphoma of presumed null-cell origin without histiocytic differentiation. The purpose of this case report is to describe a CD30+ anaplastic large-cell lymphoma of presumed null-cell origin with aberrant expression of CD13. The case illustrates the
\end{abstract}

unique usefulness of immunophenotypic and molecular techniques in establishing the correct diagnosis. The case was referred with a diagnosis of "rule out granulocytic sarcoma versus megakaryocytic malignancy" due to the morphology and a limited flow cytometric immunophenotypic ( $\mathrm{FCl}$ ) panel that had been performed and revealed expression of CD45, HLA-DR, and CD13. Subsequent morphologic review at our institution combined with an expanded $\mathrm{FCl}$ panel established the diagnosis. The differential diagnosis of a $\mathrm{CD}_{13}{ }^{+}$hematopoietic malignancy should include this entity. The prognostic significance of this finding has yet to be determined. J. Clin. Lab.Anal. 14:299-304, 2000. (๑) 2000 Wiley-Liss, Inc.

Key words: CD13; CD30+ lymphoma; flow cytometry; immunohistochemistry

The CD13 monoclonal antibody cluster recognizes a 150kilodalton glycoprotein (1). CD13 is expressed by cells within the hematopoietic system, including the majority of peripheral blood granulocytes, $25 \%$ of monocytes, and normal bone marrow cells, including approximately $40 \%$ of myeloid colony forming cells (CFU-C). CD13-positive bone marrow cells include all stages of myeloid differentiation, from the myeloblast through the granulocyte stage. CD13 is not expressed by erythroid precursors, mature erythrocytes, resting or activated B and T cells, or platelets $(2,3)$. Outside of the hematopoietic system, CD13, identical to aminopeptidase $\mathrm{N}$, is an important peptide-cleaving enzyme of the brush border membranes of the small intestine, bile duct canaliculi, renal proximal tubules, placenta, and the synaptic membranes of the central nervous system. CD13 has also been identified in fibroblasts and osteoclasts (4-6).

Due to the expression and presence of CD13 in these previously described cells, it is not surprising that CD13 is expressed by $75-95 \%$ of acute myelogenous leukemias (AML's), French-American-British (FAB) subtypes M1 through M5; $90 \%$ of cases of chronic myelogenous leukemia (CML), my- eloid blast crisis of CML, and acute myelofibrosis (2,7-11); malignancies of hepatocellular, renal cell and bile duct origin; and in a variety of benign and malignant mesenchymal tumors $(6,12,13)$. However, interestingly, CD13 is also expressed by approximately $7-10 \%$ of precursor B-cell acute lymphoblastic leukemias (ALL's), $12 \%$ of precursor T-cell ALL, some of which may represent acute mixed-lineage leukemias, and mature B-cell lymphoproliferative disorders (7,9,14-17). This latter group has included chronic lymphocytic leukemia (CLL) and its variants, the prolymphocytic variant of hairy cell leukemia (HCLV), 13\% of B-cell nonHodgkin's lymphoma (NHL) not otherwise specified, and plasma cell malignancies $(16,18)$. CD13 positivity has not been described in pure T-cell NHL's, including lymphoblastic lymphomas $(7,9)$.

*Correspondence to: Dr. Cherie H. Dunphy, Department of Pathology, St. Louis University School of Medicine, 1402 South Grand Blvd., St. Louis, MO 63104. E-mail: dunphyc@ slucare1.sluh.edu

Received 28 September 1999; accepted 28 June 2000 
We report a case of $\mathrm{CD} 30^{+}$anaplastic large-cell lymphoma of presumed null-cell origin with CD13 expression by flow cytometric immunophenotyping (FCI). The case was referred to our institution with a diagnosis of "rule out granulocytic sarcoma versus megakaryocytic malignancy" on the bases of morphology and the detection of CD45, HLA-DR, and CD13 by FCI. We report our findings.

\section{CLINICAL PRESENTATION}

An 18-year-old Caucasian male presented with a subcutaneous mass on his back that was biopsied for routine microscopic examination and submitted for FCI.

\section{MATERIALS AND METHODS}

Received from the referring outside institution was a single-cell suspension in RPMI Cellgro Medium (Fisher Scientific, St. Louis, MO) with a corresponding FCI report and a hematoxylin-eosin (HE)-stained section of the subcutaneous back lesion. A Wright's-stained cytospin preparation of the received fluid suspension was subsequently made at our institution. On the basis of cytomorphological review of the cytospin preparation, the single-cell suspension was analyzed on an Ortho Cytoronabsolute flow cytometer (Ortho Diagnostic Systems, Raritan, NJ) for various antigens using standard techniques and the following commercially available monoclonal antibodies: CD1, CD4, CD5, CD8, CD10, CD15, and HLA-DR (Ortho); CD2, CD13, CD14, CD33, CD34, CD56, CD64, and CD117 (Coulter Clone, Coulter Immunology, Hialeah, FL); CD3, CD7, and CD20 (Becton Dickinson, San Jose, CA); CD1, CD30, CD41, CD42b, $\kappa$ and $\lambda$ (Dako, Carpinteria, CA); CD45 (Caltag, Burlingame, CA); and CD138 (Biotest, Denville, NJ). Cells were gated on the basis of their forward and side light scatter properties (lymphocyte and monocyte regions). Dual staining was performed as follows: CD3/4, CD8/56, CD19/5, CD20/HLA-DR, CD45/10, CD13/14, CD33/34, CD15/117, CD41/42b, and $\kappa / \lambda$. The other antibodies were single stained.

In addition, fresh tissue from the subcutaneous back lesion was independently submitted for cytogenetic analysis to a local cytogenetics laboratory.

Subsequently, the paraffin-embedded tissue block of the subcutaneous back lesion was procured, and the following immunohistochemical (IH) stains were performed: CD30 (Nova Castra Vector Laboratories, Burlingame, CA) and CD68 (Dako Corporation, Carpinteria, CA). The IH stains were performed on the DAKO Autostainer, a universal staining system using the DAKO labeled streptavidin biotin-horse radish peroxidase (LSAB-HRP) system and DAKO 3,3'diaminobenzidine (DAB) substrate. Unmasking antigen retrieval with Citra Buffer Retrieval Solution (DAKO) was used with both stains. Control tissue included $\mathrm{CD} 30^{+}$anaplastic large-cell lymphoma (CD30) and tonsil (CD68).

\section{RESULTS}

The FCI report from the referring institution indicated that the malignant cells expressed CD45, HLA-DR, and CD13; however a full immunophenotypic panel had not been performed. The submitted diagnosis was "granulocytic sarcoma versus megakaryocytic malignancy."

Review of the provided HE-stained sections of the "back lesion" revealed several soft tissue fragments containing a diffuse infiltrate of large atypical cells with markedly irregular nuclear membranes, prominent nucleoli, and varying amounts of cytoplasm. There was a brisk mitotic rate and foci of single cell necrosis (Fig. 1).

Review of the Wright's-stained cytospin preparation of the received single-cell suspension revealed a population of predominantly large atypical cells with convoluted nuclei, prominent paranuclear clearing, prominent nucleoli, and varying

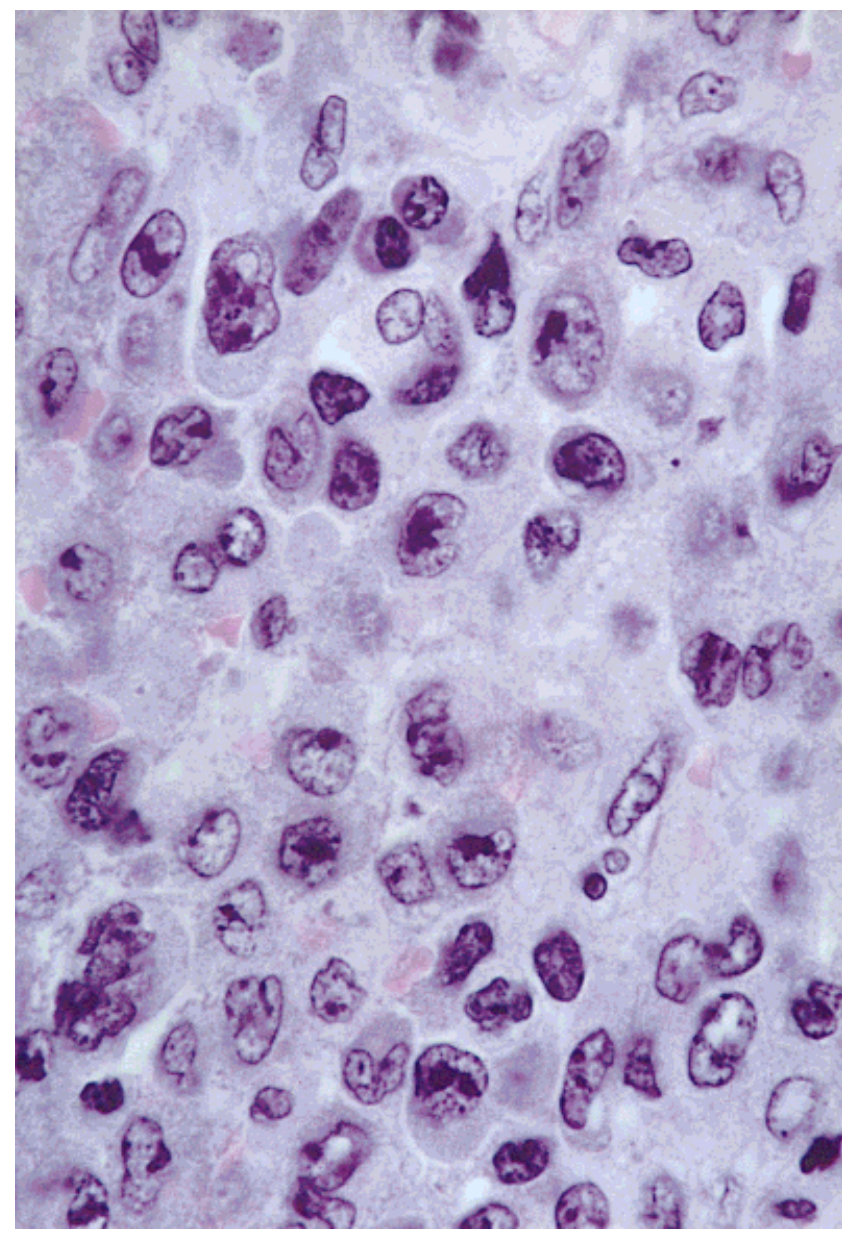

Fig. 1. Section of the "back lesion" demonstrates a diffuse infiltrate of large atypical cells with irregular nuclear membranes, prominent nucleoli, and varying amounts of cytoplasm associated with a brisk mitotic rate and single cell necrosis (hematoxylin-eosin stain, original magnification $\times 400$ ). [Color figure can be viewed in the online issue, which is available at www.interscience.wiley.com] 


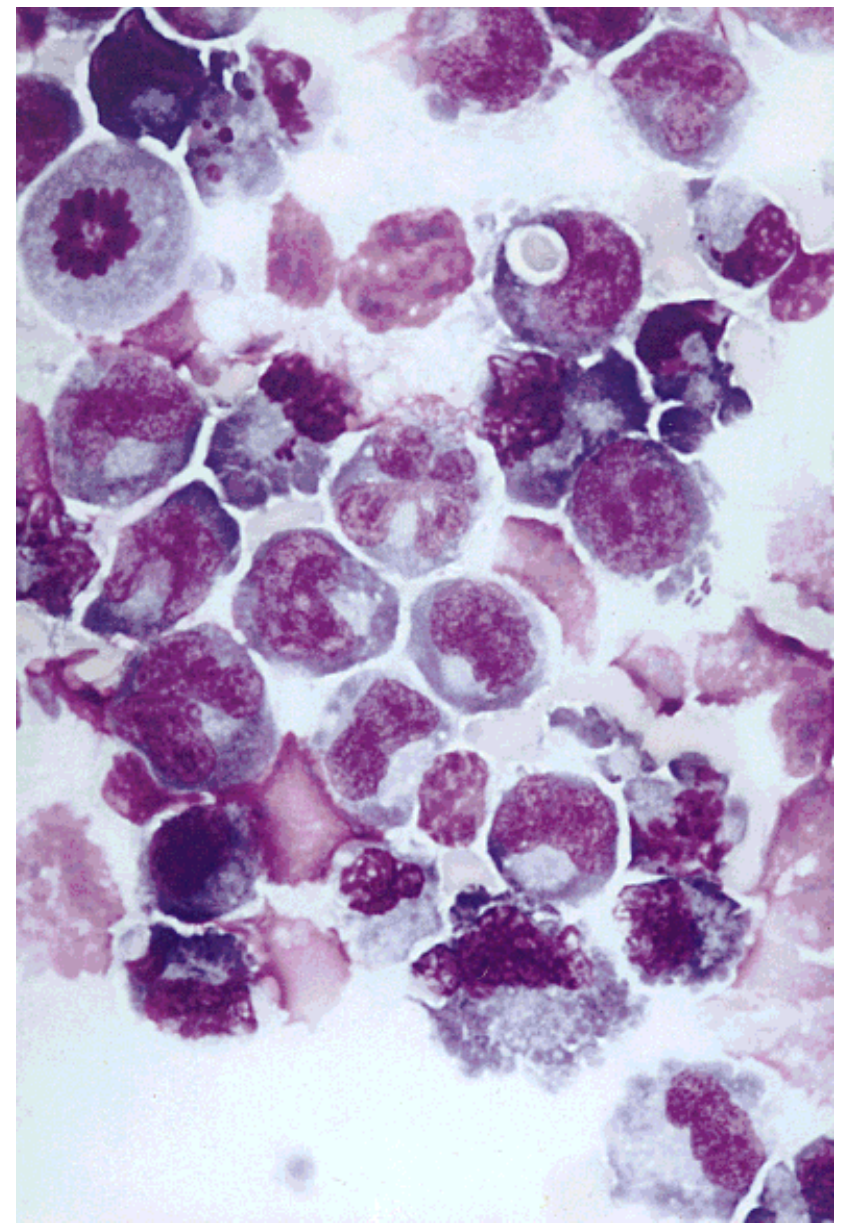

Fig. 2. Cytospin preparation of the single-cell suspension reveals the large atypical cells with convoluted nuclei, prominent paranuclear clearing, prominent nucleoli, and varying amounts of basophilic cytoplasm. Single cell necrosis is also demonstrated (Wright's stain, original magnification $\times 1,000$ ). [Color figure can be viewed in the online issue, which is available at www.interscience.wiley.com]

amounts of basophilic cytoplasm. Numerous multinucleated forms were seen (Fig. 2).

Flow cytometric analysis of the received single-cell suspension of the back lesion revealed $11 \%$ of cells within the lymphocyte region, 39\% within the monocyte (large-cell) region, and 50\% within the granulocyte region. Cells within the lymphocyte region were composed of $61 \% \mathrm{~T}$ cells (the $\mathrm{CD} 4 / \mathrm{CD} 8$ ratio is 0.22 ), $3 \% \mathrm{~B}$ cells, $8 \%$ of cells expressing the natural killer cell antigen, CD56, 3\% expressing the monocyte specific marker, CD14, and $1 \%$ expressing the human progenitor cell antigen, CD34, and the stem cell growth receptor ligand, C-kit (CD117). Cells within the monocyte (large-cell) region were composed of only $6 \%$ monocytes and approximately $92 \%$ of cells with an aberrant immunophenotype in that there was expression of CD45 (LCA), HLA-DR, CD13, and CD30 (Fig. 3). CD13 and CD30 were expressed by $92 \%$ and $97 \%$ of cells within the monocyte region, respectively; thus, co-expression
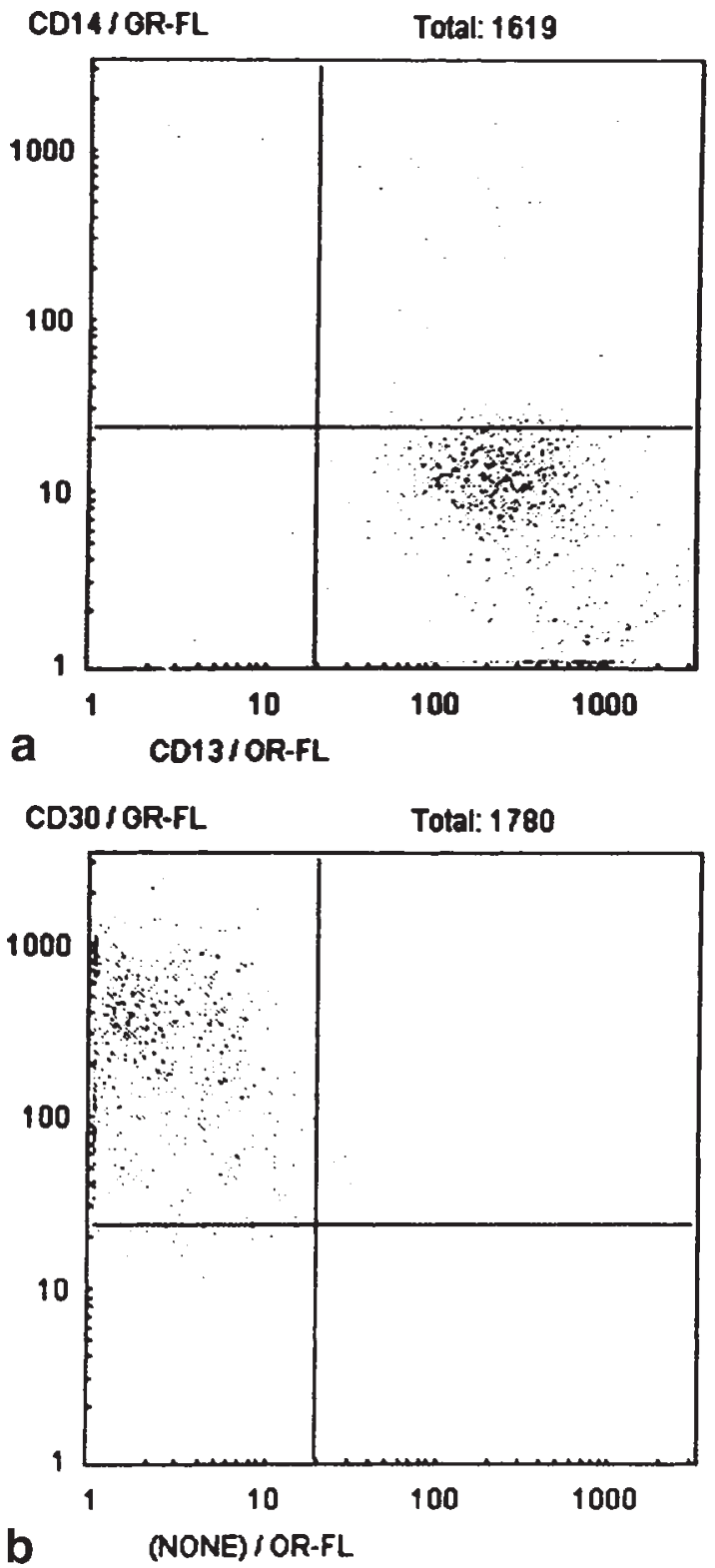

Fig. 3. Flow cytograms reveal expression of $\mathrm{CD} 13$ (a, right lower box) and CD30 (b, upper left box).

of CD13 and CD30 must have been present on cells within this region. There was no co-expression of any additional antigens analyzed by the aberrant population.

Subsequent immunohistochemical (IH) staining of the paraffin-embedded tissue was performed at the referring institution and reviewed revealing intense positivity with CD30 (Ber-H2) and ALK-1 (Fig. 4). In addition, the CD30 immunoperoxidase stain performed at our institution confirmed intense positivity of the anaplastic cells (Fig. 5); the CD68 stain did not stain the anaplastic cells.

At a later date, the local cytogenetics laboratory reported two translocations: $\mathrm{t}(2 ; 5)$ and $\mathrm{t}(1 ; 4)$ (Fig. 6). 


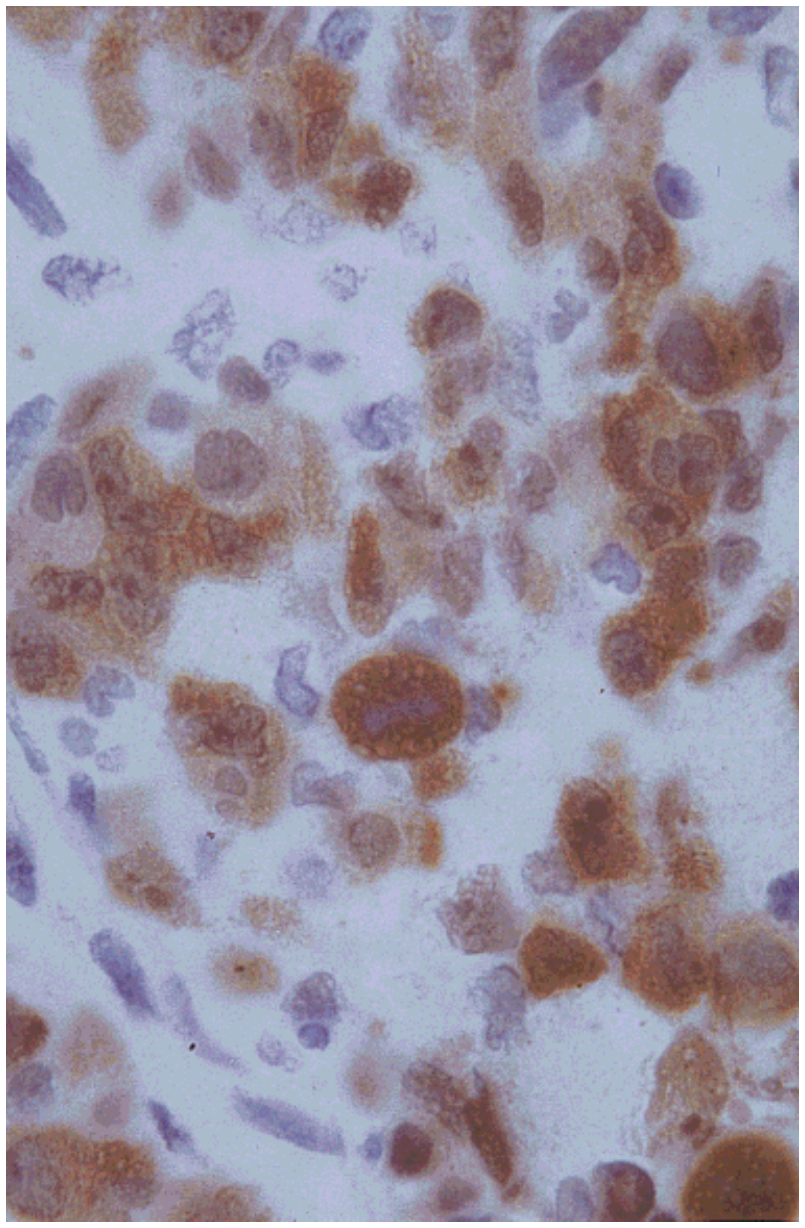

Fig. 4. ALK-1 stain performed at the referring institution reveals intense staining of the anaplastic cells (original magnification $\times 400$ ). [Color figure can be viewed in the online issue, which is available at www.interscience. wiley.com]

\section{DISCUSSION}

Within the spectrum of hematopoietic malignancies, CD13 is often or may be expressed by AML's, CML and its myeloid blast crisis, acute myelofibrosis, a subset of precursor B-cell ALL's, rare precursor T-cell ALL's, acute mixed-lineage leukemias, mature B-cell malignancies, including CLL and its variants, HCLV, B-cell NHL, and plasma cell malignancies. In addition, aberrant $\mathrm{CD} 13$ expression has rarely been described in KP-1 (CD68)-positive large-cell lymphomas (18). However, these latter cases described by Carbone et. al. also expressed CD68. The authors concluded that the study supported the view that a fraction of cases diagnosed as $\mathrm{Ki}-1(\mathrm{CD} 30)^{+}$anaplastic large-cell lymphoma represent true histiocytic lymphomas despite their $\mathrm{Ki}-1^{+}$phenotype. However, aberrant CD13 expression has not, to our knowledge, been described in pure T-cell NHL's or NHL's of null-cell origin with any evidence of histiocytic origin. In addition, there are no reports in the literature of CD30 positivity in myeloid malignancies.

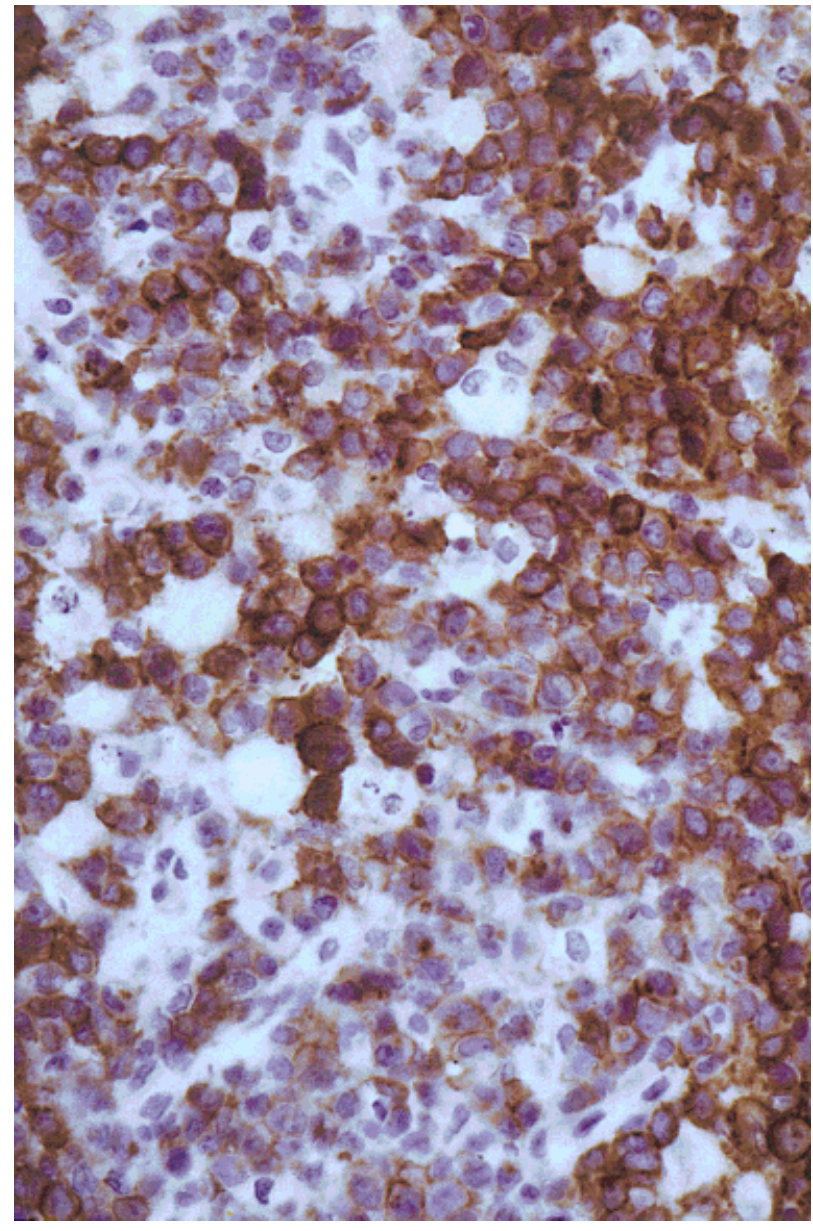

Fig. 5. CD30 stain performed at our institution confirmed intense positivity of the anaplastic cells (original magnification $\times 200$ ). [Color figure can be viewed in the online issue, which is available at www.interscience. wiley.com]

We report a case of CD30+ anaplastic large-cell lymphoma of null-cell origin with aberrant expression of CD13. The case was referred to our institution to "rule out a granulocytic sarcoma versus megakaryocytic malignancy" due to the morphology and limited FCI panel which revealed expression of CD45, HLA-DR, and CD13 by the malignancy. We reviewed the morphology and performed an expanded FCI panel. We included CD30 in our expanded FCI panel due to the morphology previously described, the patient's age, and the subcutaneous location of the mass. The nullcell origin was defined by FCI; there was not an aberrant Tcell or monoclonal B-cell population. In addition, no additional monocytic (CD14 and CD64) or myelomonocytic (CD33) antigens were detected and there was intense expression of CD30 by FCI which was confirmed by IH. In addition, the ALK-1 positivity and CD68 negativity supported a classical $\mathrm{CD} 30^{+}$anaplastic large-cell lymphoma and not a true histiocytic malignancy with a Ki-1 $1^{+}$phenotype. The de- 


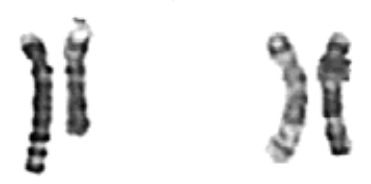

1

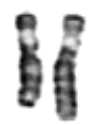

f

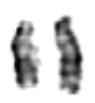

13

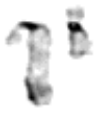

19
2

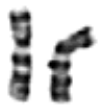

7

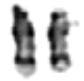

14
9

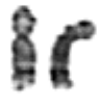

I

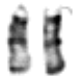

15

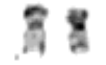

20 y
11

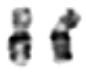

16
4

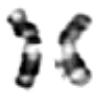

11

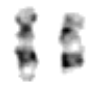

17
5

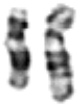

12

Result: $46, x, \operatorname{del}(X)(q 22 q 24), \operatorname{der}(2) t(2 ; 5)(p 22 ; q 35), \operatorname{der}(4) t(1 ; 4)(q 25 ; q 35), \operatorname{der}(5) t(2 ; 5)(p 22 ; q 35), \operatorname{del}(6)(q 151 q ? 21), \operatorname{add}(19)(q 13.4)[16]$

Fig. 6. G-banded karyotype shows the complex chromosome abnormalities including an interstitial deletion of the long arm of $\mathrm{X}$ chromosome (shown on the right); unbalanced translocation between the short arm of chromosome 2 and long arm of chromosome 5 resulting in derivative chro- mosomes 2 and 5; translocation between the long arms of chromosome 1 and 4; interstitial deletion of the long arm of chromosome 6 (shown on the left) and additional material on the long arm of chromosome 19.

are accrued. As mentioned previously, $\mathrm{CD} 13$ has been shown to be identical to aminopeptidase N, a membrane-bound glycoprotein thought to be involved in the process of tumor invasion (19); and thus, it would be interesting to determine if CD13 expression by this malignancy has an effect on tumor aggressiveness and behavior.

\section{REFERENCES}

1. Look AT, Peiper SC, Rebentisch MB, Ashmun RA, Roussel MF, Rettenmier CW, Sherr CJ. Transfer and expression of the gene encoding a human myeloid membrane antigen (gp150). J Clin Invest 1985;75:569-579. 
2. Griffin JD, Ritz J, Nadler LM, Schlossman SF. Expression of myeloid differentiation antigen on normal and malignant myeloid cells. J Clin Invest 1981;68:932-941.

3. Griffin JD, Ritz J, Beveridge RP, Liton JM, Daley JF, Schlossman SF. Expression of MY7 antigens on myeloid precursor cells. Int J Cell Cloning 1983;1:33-48.

4. LookAT, Ashmun RA, Shapiro LH, Peiper SC. Human myeloid plasma membrane glycoprotein CD13 (gp150) is identical to aminopeptidase N. J Clin Invest 1989;83:1299-1307.

5. Hogg N, Horton MA. Myeloid antigens: new and previously defined clusters. In: McMichael AJ, Beverley PCL, Cobbold S, et al., editors. Leucocyte typing. III. White cell differentiation antigens. Oxford: Oxford University Press; 1987, p 576-621.

6. Mechtersheimer G, Moller P. Expression of aminopeptidase N (CD13) in mesenchymal tumors. Am J Pathol 1990;137:1215-1222.

7. Griffin JD, Linch D, Sabbath K, Larcom P, Schlossman SF. A monoclonal antibody reactive with normal and leukemic human myeloid progenitor cells. Leuk Res 1984;8:521-524.

8. Drexler HG, Minowada J. The use of monoclonal antibodies for the identification and classification of acute myeloid leukemias. Leuk Res 1986;10:279-290.

9. Drexler HG, Sagawa K, Menon M, Minowada J. Phenotyping of malignant hematopoietic cells. II. Reactivity pattern of myeloid monoclonal antibodies with emphasis on MCS-2. Leuk Res 1986; 10:17-23.

10. Pombo de Oliveira MS, Matutes E, Rani S, Morilla R, Catovsky D. Early expression of MCS2 (CD13) in the cytoplasm of blast cells from acute myeloid leukemia. Acta Haematol 1988;80:61-64.

11. Look AT, Ashmun RA, Shapiro LH, et al. Report on the CD13 (aminopeptidase N) cluster workshop. In: Knapp W, Dorken B, Gilks WR, et al., editors. Leucocyte typing. IV. White cell differentiation antigens. New York: Oxford University Press; 1989, p 784-787.

12. Ortuno F, Soler J, Vilella R, Bordes R, Guanyabens C, Rubiol E, PujolMoix N. Immunophenotype of blast cells in acute myelofibrosis. Leuk Res 1990;14:849-856.

13. Finstad CL, Cordon-Cardo C, Bander NH, Whitmore WF, Melamed MR, Old LJ. Specificity analysis of mouse monoclonal antibodies defining cell surface antigens of human renal cancer. Proc Natl Acad Sci USA 1985;82:2955-2959.

14. Sobol RE, Royston I, Lebien TW, Minowada J, Anderson K, Davey FR, Cuttner J, Schiffer C, Ellison RR, Bloomfield CD. Adult acute lymphoblastic leukemia phenotypes defined by monoclonal antibodies. Blood 1985;65:730-735.

15. Stass S, Mirro J. Unexpected heterogeneity in acute leukemia: mixed lineages and lineage switch. Hum Pathol 1985;16:864-866.

16. Nakase K, Kita K, Shiku H, Tanaka I, Nasu K, Dohy H, Kyo T, Tsutani H, Kamada N. Myeloid antigen, CD13, CD14, and/or CD33 expression is restricted to certain lymphoid neoplasms. Am J Clin Pathol 1996;105:761-768.

17. Putti MC, Rondelli R, Cocito MG,Arico M, Sainata L, Conter V, Guglielmi C, Cantu-RajnoldiA, Consolini R, PessionA, Zanesco L, Masera G, Biondi A, Basso G. Expression of myeloid markers lacks prognostic impact in children treated for acute lymphoblastic leukemia: Italian experience in AIEOP-ALL 88-91 studies. Blood 1998;92(3):795-801.

18. Carbone A, Gloghini A, Volpe R, Pinto A. KP1 (CD68)-positive largecell lymphomas: a histopathologic and immunophenotypic characterization of 12 cases. Hum Pathol 1993;24(8):886-896.

19. Ohsaka A, Sato N, Imai Y, Hirai S, Oka Y, Kikuchi M, Takahashi A. Multiple gastric involvement by myeloid antigen CD13-positive nonsecretory plasma cell leukemia. Br J Haematol 1996;92:134-136. 